\title{
Photoinduced Electron Transfer Dynamics for Self-Assembled Porphyrin Arrays in Solutions and Films
}

\author{
EDUARD I. ZENKEVICH ${ }^{\mathrm{a}}$, DMITRY S. KILIN ${ }^{\mathrm{b}}$, \\ ANDREAS WILLERT ${ }^{c}$, SERGEI M. BACHILO ${ }^{a}$, ALEXANDER $^{\circ}$ \\ M. SHULGA ${ }^{\mathrm{a}}$, ULRICH REMPEL ${ }^{\mathrm{c}}$ and \\ CHRISTIAN VON BORCZYSKOWSKI ${ }^{\mathrm{c}}$ \\ ${ }^{a}$ Institute of Molecular and Atomic Physics, Acad. Sci. of Belarus, 220072 Minsk, \\ Belarus, ${ }^{\mathrm{b}}$ Belarus State University, F. Skaryna Ave. 4, 220050, Minsk, Belarus \\ and ${ }^{\mathrm{c}}$ Institut für Physik, Technische Universität Chemnitz, 09107 Chemnitz, \\ Germany
}

Electronic excitation energy deactivation in self-assembled porphyrin triads has been studied by the time correlated single photon counting technique as a function of the solvent polarity (toluene-acetone mixtures), temperature (77-350 K), and mutual spatial arrangement of the donor and acceptor subunits. The donor ( $\mathrm{Zn}$-octaethylporphyrin chemical dimer) fluorescence quenching with time constant of $1.7+10 \mathrm{ps}$ is due to competing energy migration and electron transfer processes to the acceptor (dipyridyl substituted tetrapyrrole extra-ligand). The quenching of the acceptor fluorescence (by -1.3-1.6 times) does not significantly depend on the mutual spatial arrangement of the triad subunits and increases with the solvent polarity rising and the decrease of the temperature. The obtained experimental data are analyzed using the reduced density matrix formalism in the frame of Haken-Strobl-Reineker approach taking into account the energy transfer, charge separation, and the dephasing of coherence between the excited electronic states of the triad.

Keywords: fluorescence lifetime shortening; donor-acceptor interactions; electron transfer; reduced density matrix formalism: coherence dephasing 


\section{INTRODUCTION}

At present, it is well known that the initial energy conversion in photosynthetic reaction centers is the photoinduced electron transfer (PET) from chlorophyll special pair to monomeric pheophytin [I]. Correspondingly, a number of research groups have undertaken studies of PET between large $\pi$-conjugated tetrapyrrole molecules in various model systems ${ }^{[2-4]}$. According to ${ }^{[3]}$ PET in this case can be characterized by a relatively small reorganization energy and a small inverse temperature dependence, Herein, we discuss the peculiarities of PET processes competing with the energy transfer in porphyrin triads on the basis of the experimental findings and theoretical calculations. Selfassembled triads of various but controllable geometry (Figure 1) were formed (using the extra-ligation effect ${ }^{[4]}$ ) from a covalently linked $\mathrm{Zn}$ octaethylporphyrin dimer (ZnPD) as the energy and electron donor and dipyridyl-substituted free base porphyrin $(P)$ as the acceptor.

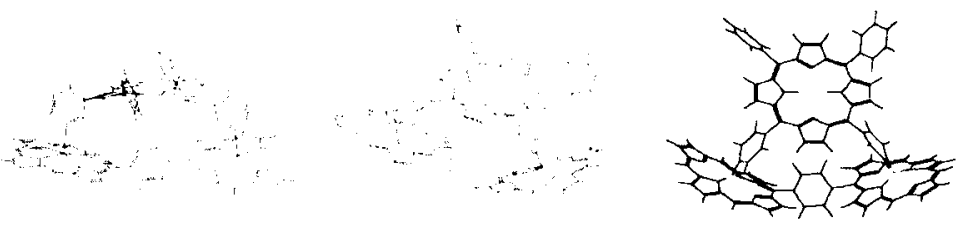

FIGURE 1 Optimized structures of porphyrin triads with various arramgement of the extra-ligand (HyperChem, release 4.0, PM3). 
The time-resolved dynamics (10 ps-nanosecond time scale) of the deactivation of the triads electronic excited states was performed with the time correlated single photon counting (TCSPC) technique.

\section{EXPERIMENTAL RESULTS}

As follows from the analysis of steady-state absorption, fluorescence and fluorescence excitation spectra of the triads reported in ${ }^{[4]}$, the $\mathrm{ZnPD}$ strong fluorescence quenching in toluene at $293 \mathrm{~K}$ is attributed to the effective dissipative singlet-singlet energy transfer $\mathrm{ZnPD} \rightarrow \mathrm{P}$ (theoretical rate constant $\mathrm{F}^{\mathrm{SS}}=3 \cdot 10^{10} \mathrm{~s}^{-1}$, Forster model). In these conditions the energy transfer may be considered as the main deactivation process for $\mathrm{ZnPD} \mathrm{S}_{1}$-state in all triads under consideration. The same situation was found for the triads in rigid polymethylmetacrylate films at $293 \mathrm{~K}$. Upon the solvent polarity increase the fluorescence excitation spectra for the triad is transformed to those for the extra-ligand only while the $\mathrm{ZnPD}$ fluorescence remains strongly quenched. It means that the sensibilization effect disappears and the other process (PET $\mathrm{ZnPD} . . \mathrm{P} \rightarrow \mathrm{ZnPD}^{+} \ldots \mathrm{P}^{-}$) becomes dominant. In addition, the quenching of the extra-ligand $P$ fluorescence intensity is observed upon the increasing polarity and the temperature lowering (Figure 2). TCSPC results obtained from a global analysis fit using three or four time constants (Figure 3 ) reveal that the amplitude spectra of the extra-ligand $\mathrm{P}$ in the triad are characterized by smaller lifetimes (6.2-7.7 ns) in toluene even with respect to those obtained for individual free bases (9.3-10.0 ns). This lifetime shortening does not significantly depend on the mutual spatial arrangement of the triad subunits (Figure 
1) but becomes essential upon the solvent polarity increase. Thus, the simultaneous decrease of the extra-ligand $\mathrm{P}$ fluorescence intensity and lifetime shortening reveal that in the triad the non-radiative deactivation of its $S_{1}$-state takes place.

\section{THEORETICAL ANALYSIS}

For the triads, the dynamics of the excited states $1=\mathrm{ZnPD}^{\circ}-\mathrm{H}_{2} \mathrm{P}$, $2=\mathrm{ZnPD}^{+}-\mathrm{H}_{2} \mathrm{P}^{-}$, and $3=\mathrm{ZnPD}-\mathrm{H}_{2} \mathrm{P}^{*}$ is described by the equation of motion for the relevant reduced density matrix ${ }^{[5.6]}$ with neglecting of the vibrational substructure of the electronic states ${ }^{[7]}$

$$
\begin{aligned}
& { }_{\partial t}^{\partial} \sigma_{\kappa \lambda}=-{ }_{\hbar}^{i}\left(\left[H_{s}, \sigma\right]_{k \lambda}+2 \delta_{k \lambda}\left\{\left[\Gamma_{\mu x}\left[n\left(\omega_{\mu \alpha}\right)+1\right]+\Gamma_{k \mu} n\left(\omega_{k \mu}\right)\right\} \sigma_{\mu \mu}\right.\right. \\
& -\sum_{\mu}\left\{\Gamma_{\mu k}\left[n\left(\omega_{\mu k}\right)+1\right]+\Gamma_{x \mu \mu} n\left(\omega_{k \mu}\right)+\Gamma_{\mu \mu}\left[n\left(\omega_{\mu \mu}\right)+1\right]+\Gamma_{\lambda \mu} n\left(\omega_{\lambda \mu}\right)\right) \sigma_{k \lambda} \\
& +\left\{\Gamma_{\lambda \dot{ }}\left[2 n\left(\omega_{\lambda \kappa}\right)+1\right]+\Gamma_{\kappa \lambda}\left[2 n\left(\omega_{k \lambda}\right)+1\right]\right\} \sigma_{\lambda \kappa} \text {. }
\end{aligned}
$$

Here the triad Hamiltonian $H_{S}$ includes the energies $E_{\lambda}$ of the corresponding states and couplings between them, $n(\omega)=\left[\exp \left(\hbar \omega / k_{\mathrm{B}} T\right)-1\right]^{-1}$ denotes Bose-Einstein distribution, $\Gamma_{\alpha d}$ the damping constant, and $\kappa, \lambda, \mu=1,2,3$. The energies $E_{1}=2.1 \mathrm{eV}$ and $E_{3}=1.91 \mathrm{eV}$ are taken from ${ }^{[4]}$. The energy of state 2 depending on the solvent polarity can be calculated using so-called Weller's formula ${ }^{[8]}$

$$
E_{2}(\varepsilon)=E_{2}\left(\varepsilon_{1}\right)+\left(\begin{array}{cc}
1 & 1 \\
\varepsilon & -\frac{1}{\varepsilon_{1}}
\end{array}\right) \underset{4 \pi \varepsilon_{0}}{e^{2}}\left(\begin{array}{c}
1 \\
2 r_{1}
\end{array}+\frac{1}{2 r_{A}}-\frac{1}{r_{i A A}}\right) .
$$

In our case the solvent mixture consists of toluene (the basic component, a static dielectric constant $\varepsilon_{1}=2.38$ ) and a small concentration $c$ of acetone (the static dielectric constant $\varepsilon_{u}=10$ ). In accordance with ${ }^{[9]}$ the effective dielectric constant of the mixture of solvents reads: 


$$
\varepsilon=\varepsilon_{1}+c^{3\left(\varepsilon_{a}-\varepsilon_{1}\right) \varepsilon_{1}} .
$$

For each parameter set we calculate the relevant reduced density matrix $\sigma_{a \lambda}(t)$ numerically. At $t=\infty$ the diagonal elements of density matrix arrive to the quasiequilibrium values. These values correspond to the fluorescence intensity of the triad subunits as presented in Figure 2.
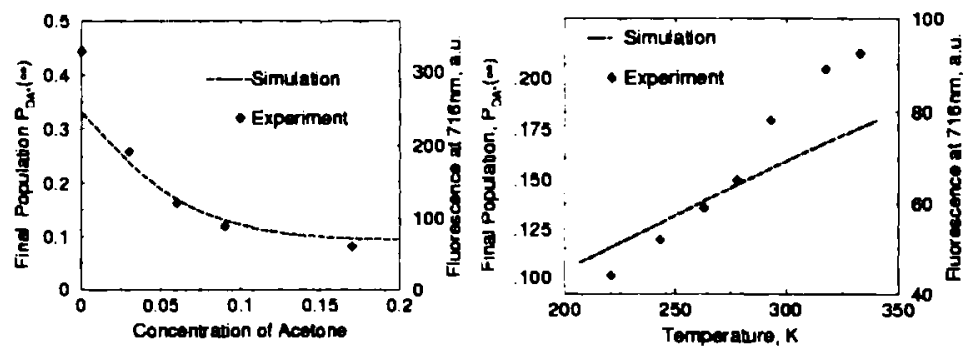

FIGURE 2. Solvent (left, $293 \mathrm{~K}$ ) and temperature (right) induced fluorescence quenching for the extra-ligand $P, C_{\text {acetone }}=7 \%$ vol.

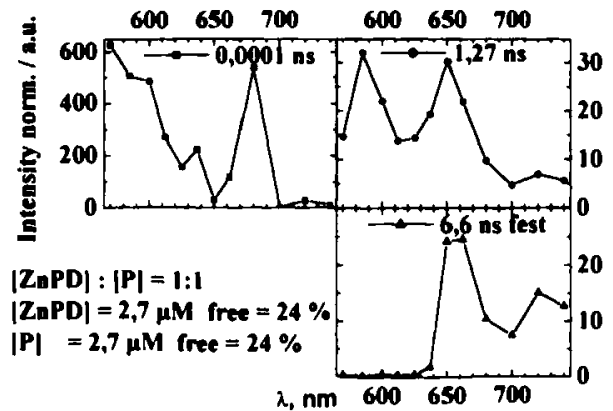

FIGURE 3. Amplitude spectra of time components of the fluorescence decay $\left(\lambda_{e x}=545 \mathrm{~nm}\right)$ for triad $\mathrm{ZnPD}-\mathrm{P}$ (toluene, $293 \mathrm{~K}$ ). 


\section{CONCLUSIONS}

It has been shown that calculated dependencies of the extra-ligand $P$ (acceptor) population in the $S_{1}$-state reflecting the intensity of its fluorescence on temperature and solvent polarity are in a reasonable accordance with experimental data. On the base of the performed experimental and theoretical investigations one concludes that the charge transfer processes induce the quenching of the singlet locally excited states of the triad. The dimer $S_{1}$-state quenching is caused by the electron transfer from $\mathrm{Zn}$-porphyrin dimer to the extra-ligand. The quenching of the extra-ligand $\mathrm{S}_{1}$-state originates from the hole transfer to the $\mathrm{Zn}$-porphyrin dimer from the extra-ligand being weakened by thermal exchange of the close lying charge transfer and extra-ligand locally excited $\mathrm{S}_{1}$-states.

\section{References}

[1] X. Hu, K. Schulten. Physics Today, 214, 28 (1997).

[2] M.R. Wasielewski. Chem. Rev, 92,435 (1992).

[3] J.M. DeGraziano, P.A. Liddell, L. Leggett, A. Moore, T. Moore, D. Gust. J. Phys. Chem., 98, 1758 (1994).

[4] S.Bachilo, A.Willert, U.Rempel, A.M.Shulga, E.I.Zenkevich, Ch. von Borczyskowski. J. Photochem. Photobiol. A: Chemistry, 126, 99 (1999).

[5] P.Reineker, Springer Tracts in Modern Physics, 94, (Springer, Berlin, 1982).

[6] P. Herman and I. Barvik, Phys. Rev. B 48, 3130 (1993).

[7] M. Schreiber, D. Kilin, U. Kleinekathoefer, J. Lumin. 83-84, 235 (1999).

[8] A. Weller, Z. Phys. Chem., Neue Folge 133, 93 (1982); Chem. Rev. 86, 403 (1986).

[9] L. Landau, Elektrodynamik der Kontinua, Akademie-Vlg., Berlin, (1985). 